\title{
Twenty-Four-Hour Intraocular Pressure Control with Omidenepag Isopropyl $0.002 \%$ in Patients with Glaucoma and Ocular Hypertension
}

\author{
Naka Shiratori ${ }^{1,2}$ \\ Yusuke Nishio ${ }^{1,2}$ \\ Ayaka Takeda ${ }^{1,2}$ \\ Shio Sugimoto ${ }^{1,2}$ \\ Kenji Takazawa ${ }^{2}$ \\ Naomi Otsuka ${ }^{3}$ \\ Naruhiro Ishida ${ }^{3}$ \\ Daisuke Shii ${ }^{3}$ \\ Kiyotaka Hori ${ }^{3}$ \\ Kenji Nakamoto' \\ 'Department of Ophthalmology, Nippon \\ Medical School, Tokyo, Japan; \\ ${ }^{2}$ Shinanozaka Clinic, Tokyo, Japan; ${ }^{3} J a p a n$ \\ Medical Affairs Group, Santen \\ Pharmaceutical Co. Ltd., Osaka, Japan
}

Correspondence: Naka Shiratori Department of Ophthalmology, Nippon Medical School, I-I-5 Sendagi, Bunkyo-ku, Tokyo, Japan

Tel $+8|-3-3822-2| 3 \mid$

Fax +8I-3-5685-0988

Email shiratori@nms.ac.jp
Purpose: To clarify the intraocular pressure (IOP)-lowering effect of a selective prostanoid EP2 receptor agonist, omidenepag isopropyl (OMDI) during a 24-hour period.

Patients and Methods: Subjects aged $\geq 20$ years and with diagnosed, untreated primary open-angle glaucoma or ocular hypertension were enrolled. IOP measurements were performed every 4 hours over a 24-hour period using a Goldmann applanation tonometer (GAT) and Icare PRO tonometer (PRO). The baseline 24-hour IOP was measured in untreated subjects. After the baseline measurements, participants were given OMDI 1 drop once daily at night for 4 weeks. At week 4, the IOP measurement was repeated under the same conditions. Diurnal ( 9 am, 1 pm, 5 pm) and nocturnal (9 pm, 1 am, 5 am) IOP measurements were compared between baseline and treatment with OMDI. Safety measures included adverse events, slit-lamp biomicroscopy, visual acuity, heart rate and blood pressure.

Results: Of 27 participants enrolled, 25 patients (20 males and 5 females, average age 52.2 \pm 8.5 years) completed the study. In the sitting position, the baseline diurnal and nocturnal mean IOPs (GAT) were $19.1 \pm 2.1 \mathrm{mmHg}$ and $18.2 \pm 2.6 \mathrm{mmHg}$, respectively, the diurnal and nocturnal mean IOP reduction from baseline were $-2.8 \pm 2.6 \mathrm{mmHg}(\mathrm{p}<0.0001)$ and $3.3 \pm 2.9 \mathrm{mmHg}(\mathrm{p}<0.0001)$, respectively, mean 24-hour IOP (GAT) was significantly lower with the OMDI treatment $(-3.1 \pm 2.5 \mathrm{mmHg}, \mathrm{p}<0.0001)$. In the supine position, the baseline nocturnal mean IOP (PRO) was $17.99 \pm 2.22 \mathrm{mmHg}$, and the nocturnal mean IOP reduction from baseline was $-1.78 \pm 2.37 \mathrm{mmHg}(\mathrm{p}=0.0009)$ after 4 weeks of the treatment. Nine adverse events were observed in 8 patients including mild conjunctival hyperemia $(n=8)$ and mild iritis $(n=1)$. There were no significant effects on systemic safety.

Conclusion: Once daily OMDI treatment was able to produce stable 24-hour IOP reduction. Keywords: omidenepag isopropyl, prostanoid EP2 agonist, 24-hour IOP control

\section{Introduction}

Elevated intraocular pressure (IOP) is a major risk factor for the progression of glaucoma. Lowering IOP is the only therapeutic approach to reduce the risk of glaucomatous vision loss. ${ }^{1,2}$ However, IOP is well-known to fluctuate widely over a 24-hour period. ${ }^{3}$ Therefore, in order to achieve optimal IOP control through appropriate therapeutic intervention, it is important to understand the IOP daily variation pattern of individual patients and to provide treatment based on the characteristics of the drug. ${ }^{4}$

While all approved glaucoma medications have proven IOP-lowering effects in the daytime, the effectiveness of some medicines are affected by circadian rhythms. For 
example, $\alpha$-agonist and $\beta$-blocker have reduced or no IOPlowering effects during nocturnal periods. ${ }^{5,6}$ Prostanoid FP receptor agonists sustain IOP reduction nocturnally, although a reduced magnitude versus daytime reductions. ${ }^{7,8}$

Recently, the prostanoid EP2 receptor agonist, omidenepag isopropyl $0.002 \%$ (OMDI), has emerged as a novel treatment option in patients with glaucoma and ocular hypertension. OMDI was approved for the reduction of IOP in patients with glaucoma in Japan in November 2018. ${ }^{9-13}$ OMDI appears to provide IOP reductions comparable to those of prostaglandin F2 $\alpha$ analogues without the cosmetic side effects common to prostaglandin F2 $\alpha$ analogues especially prostaglandin-associated periorbitopathy (PAP) syndrome. ${ }^{14}$ However, there are no published data on the 24-hour efficacy of the prostanoid EP2 agonist omidenepag especially during nocturnal periods.

The purpose of this study was to examine the intraocular pressure lowering effect of the prostanoid EP2 agonist OMDI during a 24-hour period in patient with glaucoma or ocular hypertension.

\section{Materials and Methods}

Study Design, Setting, and Trial

\section{Registration}

This prospective, open-label, experimental study was approved by the Certified Review Board of the Medical Corporation Shinanokai, Shinanozaka Clinic, was conducted in accordance with the tenets of the Declaration of Helsinki and Clinical Trials Act in Japan, and was registered as a clinical trial (Japan Registry of Clinical Trials: jRCT, ID: jRCTs031200106). All participants were fully informed of the purpose and procedures of this study, and all provided written consent to the investigators. This study was conducted from September 2020 to January 2021 at Shinanozaka Clinic (Tokyo, Japan).

\section{Participants}

All subjects enrolled in this study were patients with primary open-angle glaucoma (POAG) or ocular hypertension $(\mathrm{OH})$ in at least one eye who had not been treated within 4 weeks before enrollment, who had an IOP of 18 $\mathrm{mmHg}$ or more for POAG, $21 \mathrm{mmHg}$ or more for $\mathrm{OH}$ in at least one eye, and $34 \mathrm{mmHg}$ or less in both eyes, and who were aged 20 years and old.

The exclusion criteria were as follows: aphakic or pseudophakic eyes, any corneal abnormalities or other disorders that may interfere with accurate tonometry with the Goldmann Applanation Tonometer (GAT), a history of corneal refractive surgery, corrected visual acuity of $20 / 33$ or less, the possibility of developing visual field impairment during the study period, severe visual field impairment within 6 months including the date of consent acquisition (eg, $-20 \mathrm{~dB}$ or less with a mean deviation in the Humphrey visual field test program central 30-2), active external eye disease, eye/eyelid inflammation, and infections, a history of anterior or internal eye surgery, a history of glaucoma surgery including laser treatment, hypersensitivity to the components of the drug used in this study, being pregnant, a lactating woman, possibly pregnant, wishing to become pregnant during the study period, or unable to use proper contraception during the study period.

\section{Procedure}

During the study period, each patient routinely underwent a comprehensive clinical examination that included measuring IOP with a GAT (AT-900, Haag-Streit Diagnostics) and Icare PRO tonometer (Icare Finland Oy, Espoo, Finland) (PRO), slit-lamp biomicroscopy, fundoscopy, blood pressure determination, and heart rate measurement. In addition, gonioscopy were conducted at the time of enrollment, and the central corneal thickness was measured at screening visit and efficacy visit (4 weeks after treatment) once each time. Visual acuity tests were conducted at the time of enrollment and the end of the study.

Baseline IOP was measured in the sitting position with the PRO and then with the GAT; a total of 6 measurements were made at 4-hour intervals (nocturnal period: $9 \mathrm{pm}, 1$ am and 5 am, diurnal periods: 9 am, $1 \mathrm{pm}$ and $5 \mathrm{pm}$ ). In addition, IOP was measured in the supine position with the PRO at $9 \mathrm{pm}, 1$ am and 5 am after the measurements in the sitting position. The maximum acceptable variation in the time of the IOP measurement was \pm 30 minutes. Blood pressure and heart rate were also measured at the same times as the IOP measurement. After the baseline measurements, participants received OMDI (EYBELIS $^{\circledR}$ ophthalmic solution $0.002 \%$, Santen Pharmaceutical Co., Ltd., Japan) 1 drop once daily at night for 4 weeks. For patients with bilateral POAG or $\mathrm{OH}$ with both eyes meeting the eligibility criteria, the eye with the higher mean 24hour IOP at baseline was selected as the study eye; if the IOP was equal in both eyes, the right eye was selected for this study.

At each time point, IOP was measured three times with the GAT and the median value adopted for the analysis. 
With the PRO, the measurement was made at least six times until "deviation OK" was displayed on the tonometer's screen, and the mean of these values was adopted. On the day before hospitalization for the 24hour IOP measurement, the patients were asked to instill the eye drops between $8 \mathrm{pm}$ and $10 \mathrm{pm}$. On the days of hospitalization, the eye drops were administered at $9 \mathrm{pm}$ after the measurements of IOP, blood pressure, and heart rate.

\section{Outcomes}

Primary outcome measure was the comparison of change in the diurnal and nocturnal mean IOP between the OMDI treatment and the baseline. Secondary outcome measures were the comparison of change in IOP at each time point of measurement, change in 24-hour mean IOP, 24-hour IOP fluctuation range, peak and minimum IOP, ocular perfusion pressure (OPP), blood pressure, heart rate, central corneal thickness, and visual acuity between the treatment and the baseline. The sitting OPP was calculated using a previously described formulae as follows: ${ }^{15}$ OPP $=2 / 3 \times$ mean blood pressure - IOP.

\section{Statistical Analysis}

The backgrounds data and adverse event profiles of all the enrolled patients, and the other data for all the patients who completed the study, were analyzed using SAS software version 9.4 (SAS Institute Inc., Cary, NC, USA). Statistical comparisons between the OMDI treatment and baseline values were performed for each parameter using the paired $t$-test. The significance level was 5\% (twosided).

\section{Results}

Of the 27 subjects enrolled, 25 completed the study. All participants were diagnosed as having POAG or $\mathrm{OH}$, and were untreated before the enrolment. The data on subjects' demographic characteristics data are presented in Table 1. Two subjects were discontinued from the study because of protocol violations. Therefore, the safety population comprised $27 \mathrm{sub}-$ jects who received more than one dose of OMDI.

Figure 1 shows the 24-hour sitting IOP profiles using the GAT at baseline and under treatment with OMDI. The baseline mean IOP appeared higher during the diurnal period than during nocturnal period. During the 24-hour periods of observation, the mean IOP was consistently lower under OMDI treatment than at the baseline. The sitting peak IOP and minimum IOP during the 24-hour
Table I Demographic Characteristics of Study Paticipants

\begin{tabular}{|l|c|}
\hline Gender & 20 male, 5 female \\
Age (years) & $52.2 \pm 8.5(40-73)$ \\
Diagnosis & 18 POAG, $7 \mathrm{OH}$ \\
Central cornea thickness $(\mu \mathrm{m})$ & $545.2 \pm 42.2(454-623)$ \\
Systolic blood pressure $(\mathrm{mmHg})$ & $121.2 \pm 13.7(95-144)$ \\
Diastolic blood pressure $(\mathrm{mmHg})$ & $77.1 \pm 10.0(55-99)$ \\
Heart rate (beats/min) & $70.6 \pm 8.4(53-89)$ \\
\hline
\end{tabular}

Notes: Value represents the mean \pm standard deviation (range). Blood pressure and heart rate data show average for 24 hours in sitting position.

Abbreviations: POAG, primary open angle glaucoma; $\mathrm{OH}$, ocular hypertension.

period was significantly decreased under OMDI treatment by $-3.3 \pm 3.1 \mathrm{mmHg}$ and $-3.1 \pm 2.7 \mathrm{mmHg}$, respectively $(\mathrm{p}<0.0001)$. In addition, the 24-hour sitting IOP fluctuation at baseline and under OMDI treatment was $4.8 \pm 1.3$ $\mathrm{mmHg}$ and $4.6 \pm 2.2 \mathrm{mmHg}$, respectively. There were no significant differences in the fluctuation range before and after treatment.

Figure 2 presents the profiles of 24-hour habitual IOP (sitting during the diurnal period and supine during the nocturnal period) using the Icare PRO tonometer at baseline and under OMDI treatment. The baseline IOP seemed to be higher during the nocturnal period than during the diurnal period. The habitual IOP under OMDI treatment was significantly lower than the baseline IOP except at 5 am and $5 \mathrm{pm}$.

Table 2 summaries the mean diurnal and nocturnal IOP values under the two experimental conditions. In the sitting IOP measurements using the GAT, the diurnal and nocturnal mean IOP under the OMDI treatment were significantly lower than the corresponding diurnal and nocturnal baseline IOP ( $<<0.0001)$. In the habitual IOP measurement using the $\mathrm{PRO}$, the sitting diurnal and supine nocturnal mean IOP under OMDI treatment were also significantly lower than the baseline IOP $(\mathrm{p}=0.0010, \mathrm{p}=0.0009)$.

Table 3 shows OPP values in the sitting position under OMDI treatment (with GAT IOP data used for calculation). The mean OPP under OMDI treatment was significantly greater than the baseline during the diurnal periods $(\mathrm{p}=0.0032)$, and nocturnal periods $(\mathrm{p}=0.0001)$. OMDI treatment had no significant effect on the systemic blood pressure and heart rate in the both sitting and supine positions during diurnal and nocturnal periods.

Nine adverse events were observed in 8 patients in this study including mild conjunctival hyperemia $(\mathrm{n}=8)$ and mild iritis $(n=1)$, which were disappeared by the end of treatment. The central corneal thickness during the study period was significantly increased by $18.0 \pm 16.2 \mu \mathrm{m}$ under the OMDI 


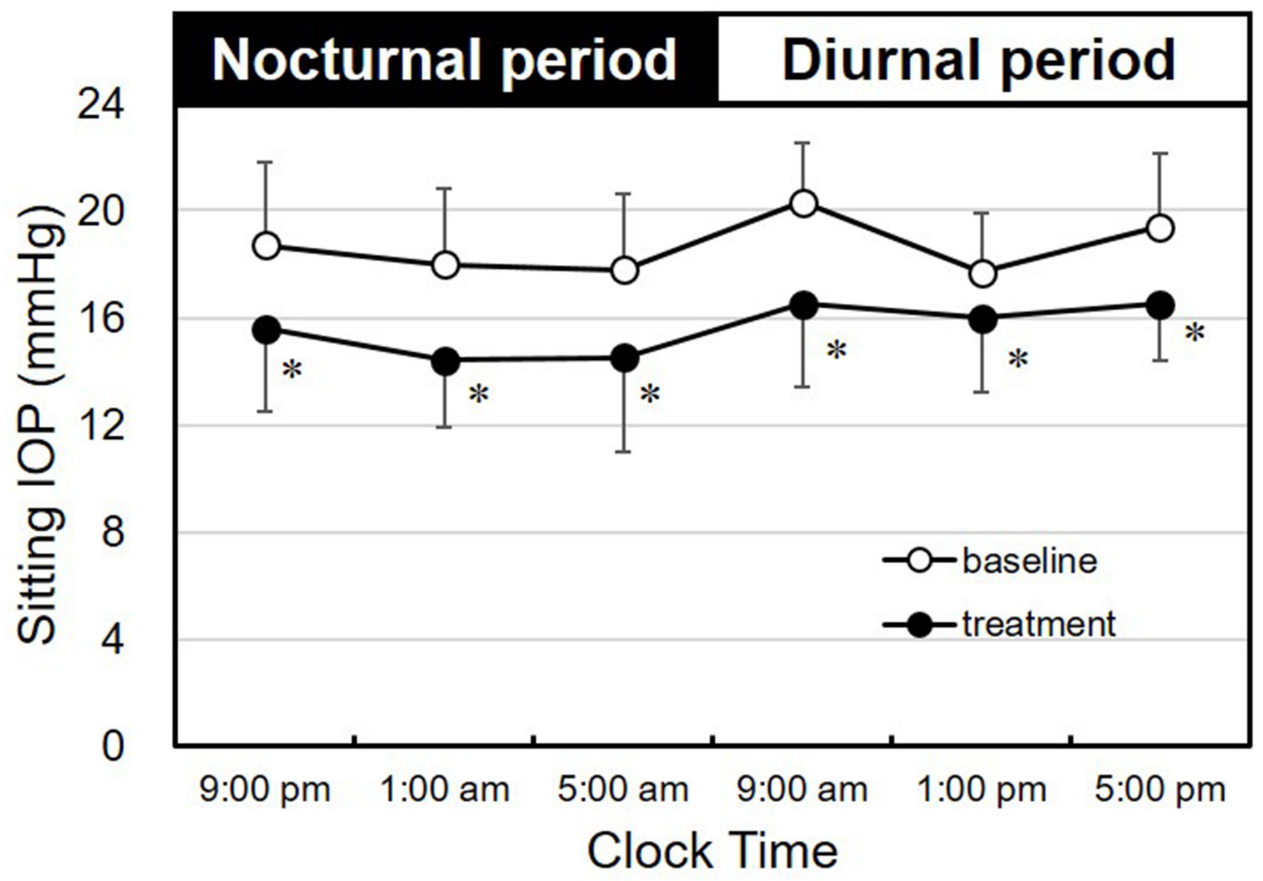

Figure I Effect of omidenepag isopropyl on 24-hour IOP (sitting position, Goldmann applanation tonometer). Sitting IOP measurements were taken from 25 patients during 24 -hour period. The baseline is indicated by open circles, and omidenepag isopropyl $0.002 \%$ treatment for 4 weeks by closed circles. Values are presented as the mean \pm standard deviation. ${ }^{*} \mathrm{P}<0.01$ vs the baseline by paired $t$-test.

Abbreviation: IOP, intraocular pressure.

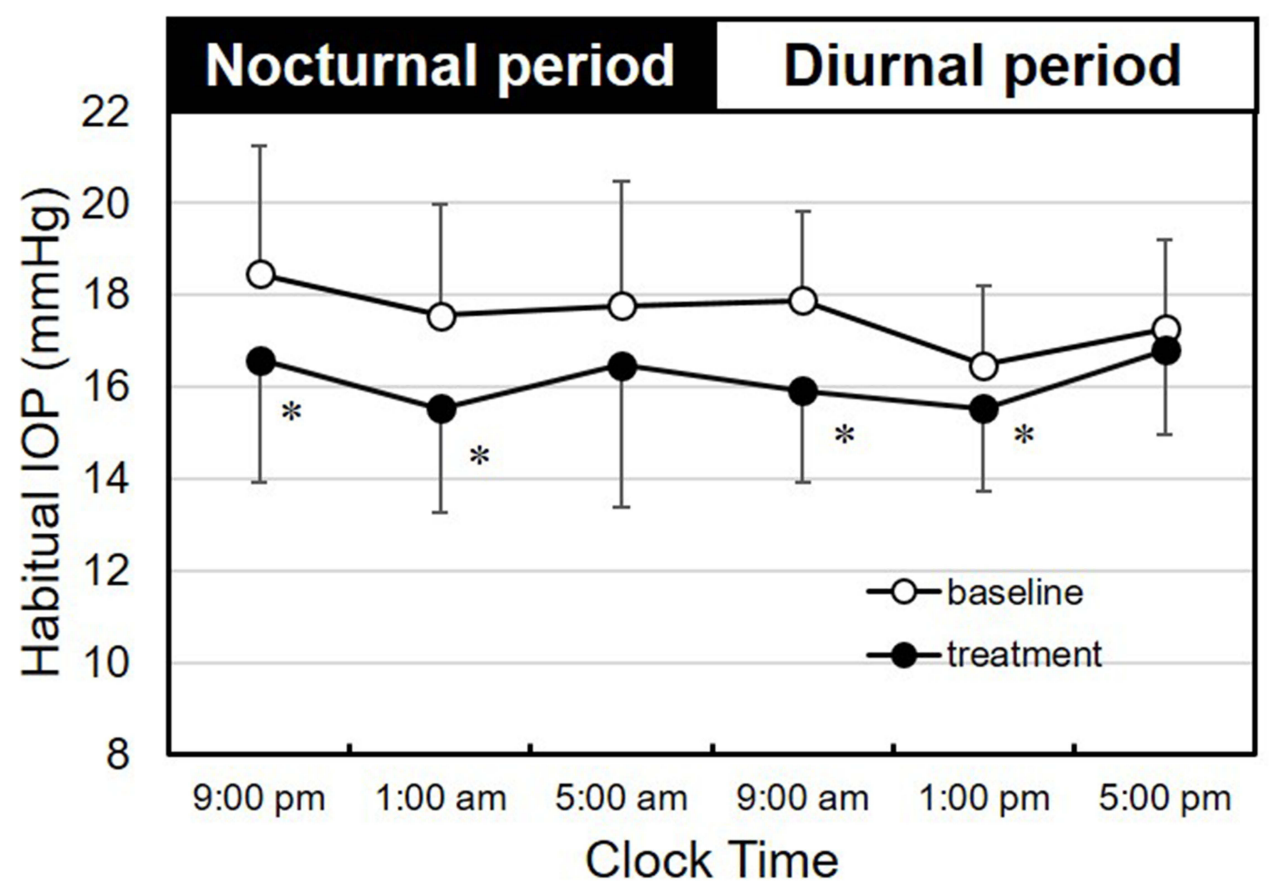

Figure 2 Effect of omidenepag isopropyl on 24-hour IOP (habitual position, Icare PRO tonometer). IOP measurements were taken from 25 patients habitual position (sitting during the diurnal period and supine during the nocturnal period). Data were from the same subjects as in Figure I. The baseline is indicated by open circles, and omidenepag isopropyl $0.002 \%$ treatment for 4 weeks by closed circles. Values are presented as the mean \pm standard deviation. $* \mathrm{P}<0.05$ vs baseline by paired $t$-test. Abbreviation: IOP, intraocular pressure. 
Table 2 Mean IOP Before and After Treatment with Omidenepag Isopropyl 0.002\%

\begin{tabular}{|l|c|c|c|c|}
\hline & Baseline $\mathbf{( m m H g})$ & Treatment $\mathbf{( m m H g})$ & Difference $\mathbf{( m m H g )}$ & p value \\
\hline Sitting IOP (GAT) & & & $-2.8 \pm 2.6$ & $<0.000 \mathrm{I}$ \\
Diurnal Mean IOP & $19.1 \pm 2.1$ & $16.3 \pm 2.4$ & $-3.3 \pm 2.9$ & $<0.000 \mathrm{I}$ \\
Nocturnal Mean IOP & $18.2 \pm 2.6$ & $14.8 \pm 2.6$ & $-3.1 \pm 2.5$ & $<0.000 \mathrm{I}$ \\
24-hour Mean IOP & $18.7 \pm 2.2$ & $15.6 \pm 2.4$ & & \\
\hline Habitual IOP (Icare PRO) & & & $-1.12 \pm 1.49$ & 0.0010 \\
Diurnal Mean IOP (sitting) & $17.21 \pm 1.67$ & $16.08 \pm 1.44$ & $-1.78 \pm 2.37$ & 0.0009 \\
Nocturnal Mean IOP (supine) & $17.99 \pm 2.22$ & $16.20 \pm 2.27$ & \\
\hline
\end{tabular}

Notes: All data represent the mean \pm standard deviation $(\mathrm{N}=25)$. Diurnal points: 9 am, I pm, and 5 pm. Nocturnal points: 9 pm, I am, and 5 am. Habitual IOP: sitting during the diurnal period and supine during the nocturnal period. $\mathrm{P}$ value performed with paired $t$-test.

Abbreviations: GAT, Goldmann applanation tonometer; Icare PRO, Icare PRO tonometer; IOP, intraocular pressure.

Table 3 Mean OPP Before and After Treatment with Omidenepag Isopropyl 0.002\%

\begin{tabular}{|l|c|c|c|c|}
\hline & Baseline (mmHg) & Treatment $\mathbf{( m m H g )}$ & Difference (mmHg) & p value \\
\hline Diurnal Mean OPP & $41.7 \pm 7.6$ & $44.8 \pm 7.7$ & $3.0 \pm 4.7$ & 0.0032 \\
Nocturnal Mean OPP & $43.4 \pm 7.3$ & $47.1 \pm 6.6$ & $3.7 \pm 4.0$ & 0.0001 \\
24-hour Mean OPP & $42.6 \pm 7.2$ & $45.9 \pm 7.0$ & $3.4 \pm 3.9$ & 0.0002 \\
\hline
\end{tabular}

Notes: Each value represents the mean \pm standard deviation. Diurnal points: 9 am, I pm, and 5 pm. Nocturnal points: 9 pm, I am, and 5 am. P value performed with paired $t$-test.

Abbreviation: OPP, ocular perfusion pressure.

treatment $(\mathrm{p}<0.0001)$. There were no significant effects on visual acuity, slit-lamp biomicroscopy, and systemic safety.

\section{Discussion}

This study investigated the 24-hour IOP-lowering effect of OMDI, the world's first launched selective prostanoid EP2 receptor agonist, in patients with glaucoma or ocular hypertension. The results showed a significant IOP-lowering effect of OMDI not only during the daytime but also at night time. Moreover, the attenuation of the nocturnal IOPlowering effect observed with other glaucoma $\operatorname{drugs}^{5-8}$ is characteristically not observed with OMDI. In addition, omidenepag significantly reduced peak IOP and minimum IOP during the 24-hour period of observation despite the variable onset time which differed for each patient, suggesting that OMDI can stably control IOP over a 24-hour period.

The IOP is known to vary greatly over a 24 -hour period, and to be influenced by the patient's daily activities and posture. ${ }^{16,17}$ Many patients experience peak IOP outside of clinic hours, and peak IOPs occur, not uncommonly, at nighttime. ${ }^{18}$ Therefore, in order to achieve optimal IOP control for the patient, it may be necessary to acquire an individual's daytime IOP pattern information in a highly accurate and standardized manner (eg, in the habitual or sitting position). On the other hand, it is known that the effects of IOP-lowering agents are not uniform for
24 hours. For example, beta-blockers, alpha-2 agonists, some prostanoid FP receptor agonists, and combinations of these agents may not be appropriate for patients with nocturnal peak IOPs because they attenuate nocturnal IOPlowering effects. ${ }^{4}$ Since it is difficult to accurately assess 24-hour IOP patterns in all glaucoma patients before prescribing, the prostanoid EP2 receptor agonist omidenepag, which has less fluctuation in drug efficacy during the day, may be useful as a first-line treatment for new patients.

OMDI significantly increased OPP, suggesting that it may also provide the benefit of improved ocular circulation. Generally, decreased ocular circulation has been reported as a risk factor for the development of glaucoma and progression of visual field disorders, ${ }^{16}$ but the effect of OMDI on the OPP and whether it will actually have a positive effect are unclear at this time, and further investigation is required.

Adverse reactions due to OMDI such as conjunctival hyperemia and iritis occurred in this study, but all events were mild and disappeared after withdrawal of the drug. In addition, no systemic adverse reactions were observed, and the drug was considered to be well tolerated. Although a significant increase in central corneal thickness of around $20 \mu \mathrm{m}$ was observed after administration of OMDI, there were no obvious changes in the visual acuity of the patients, suggesting that the effects on daily life were minimal. Recently, it has been reported that prostaglandin-related drugs cause thinning 
of the central cornea and reduction of corneal stiffness, which may result in overestimation of the IOP-lowering effect, ${ }^{19,20}$ while corneal thickening by OMDI may lead to underestimation of the IOP-lowering effect. Thus, additional studies such as IOP monitoring during long-term use are required.

There are several limitations to this study. First, since this study has no comparison group such as placebo or positive control group, there may be a bias in the evaluation of the IOP-lowering effect of OMDI. Second, a small number of patients were studied at a single institution, suggesting that the reproducibility should be verified. These issues need further investigation.

In conclusion, once daily OMDI treatment was able to provide stable 24-hour IOP reduction, indicating that the prostanoid EP2 receptor agonist should be considered a new treatment option for patients with glaucoma and ocular hypertension.

\section{Data Sharing Statement}

The datasets analyzed during this study are available from the corresponding author on reasonable request.

\section{Acknowledgments}

The author thanks to Mr. Kazunori Santo who provided in the technical assistance. This study was sponsored by Santen Pharmaceutical Co., Ltd.

\section{Disclosure}

The authors have made the following disclosure: NS, YN, AT, SS and KT received non-financial research support from Santen Pharmaceutical Co., Ltd. during the conduct of study; NO, NI, DS, and KH were employees of Santen Pharmaceutical Co., Ltd.; KN had a consulting relationship with Santen Pharmaceutical Co., Ltd and reports personal fees from Shinanozaka Clinic. The authors report no other conflicts of interest in this work.

\section{References}

1. Heijl A, Leske M, Bengtsson B, et al.; Early manifest glaucoma trial group. Reduction of intraocular pressure and glaucoma progression: result from the early manifest glaucoma trial. Arch Ophthalmol. 2002;120(10):1268-1279. doi:10.1001/archopht.120.10.1268

2. Leske M, Heijl A, Hussein M, et al. Factors for glaucoma progression and the effect of treatment: the early manifest glaucoma trial. Arch Ophthalmol. 2003;121(1):48-56. doi:10.1001/archopht.121.1.48

3. Kass MA, Heuer DK, Higginbotham EJ, et al. The ocular hypertension treatment study: a randomized trial determines that topical ocular hypotensive medication delays or prevents the onset of primary open glaucoma. Arch Ophthalmol. 2002;120(6):701-713. doi:10.1001/ archopht.120.6.701
4. Mansouri K, Tanna AP, DeMoraes CG, et al. Review of the measurement and management of 24-hour intraocular pressure in patients with glaucoma. Surv Ophthalmol. 2020;65:171-186. doi:10.1016/j. survophthal.2019.09.004

5. Liu JHK, Medeiros FA, Slight JR, et al. Diurnal and nocturnal effects of brimonidine monotherapy on intraocular pressure. Ophthalmology. 2010;117(11):2075-2079. doi:10.1016/j.ophtha.2010.03.026

6. Gulati V, Fan S, Zhao M, et al. Diurnal and nocturnal variations in aqueous humor dynamics of patients with ocular hypertension undergoing medical therapy. Arch Ophthalmol. 2012;130(6):677-684. doi:10.1001/archophthalmol.2011.2573

7. Orzalesi N, Rossetti L, Bottoli A, et al. Comparison of the effects of latanoprost, travoprost, and bimatoprost on circadian intraocular pressure in patients with glaucoma and ocular hypertension. Ophthalmology. 2006;113(2):239-246. doi:10.1016/j.ophtha.2005.10.045

8. Oddone F, Rossetti L, Tanga L, et al. Effects of topical bimatoprost $0.01 \%$ and timolol $0.5 \%$ on circadian IOP, blood pressure and perfusion pressure in patients with glaucoma or ocular hypertension: a randomized, double masked, placebo-controlled clinical trial. PLoS One. 2015;10(10):e0140601. doi:10.1371/journal.pone.0140601

9. Aihara M, Lu F, Kawata H, et al. Omidenepag isopropyl versus latanoprost in primary open-angle glaucoma and ocular hypertension: the Phase 3 AYAME study. Am J Ophthalmol. 2020;220:53-63. doi:10.1016/j.ajo.2020.06.003

10. Aihara M, Lu F, Kawata H, et al. Twelve-month efficacy and safety of omidenepag isopropyl, a selective EP2 agonist, in open-angle glaucoma and ocular hypertension: the RENGE study. Jpn J Ophthalmol. 2021:1-10. doi:10.1007/s10384-021-00868-y

11. Aihara M, Ropo A, Lu F, et al. Intraocular-pressure lowering effect of omidenepag isopropyl in latanoprost non-/low-responder patients with primary open-glaucoma or ocular hypertension: the FUJI study. Jpn J Ophthalmol. 2020;64(4):398-406. doi:10.1007/s10384020-00748-x

12. Inoue K, Inoue J, Kunimatsu-Sanuki S, et al. Short-term efficacy and safety of omidenepag isopropyl in patients with normal tension glaucoma. Clin Ophthalmol. 2020;14:2943-2949. doi:10.2147/OPTH. S271789

13. Nakakura S, Kanamori A, Fukuma Y, et al. Evaluation of early medication persistence with omidenepag isopropyl, a topical selective prostaglandin EP2 agonist, in patients with glaucoma: a retrospective two-institute study. BMJ Open. 2021;11(1):e040301. doi:10.1136/ bmjopen-2020-040301

14. Aihara M, Aung T, Bacharach J, et al. Omidenepag isopropyl ophthalmic solution for open-angle glaucoma and ocular hypertension: an update. Expert Rev Ophthalmol. 2021;16(4):243-250. doi:10.1080/17469899.2021.1935241

15. Okuno T, Sugiyama T, Kohyama M, et al. Ocular blood flow changes after dynamic exercise in humans. Eye. 2006;20:796-800. doi:10. 1038/sj.eye.6702004

16. Quatanta L, Katsanos A, Russo A, et al. 24-hour intraocular pressure and ocular perfusion pressure in glaucoma. Surv Ophthalmol. 2013;58(1):26-41. doi:10.1016/j.survophthal.2012.05.003

17. Liu JHK, Kripke DF, Twa MD, et al. Twenty-four-hour pattern of intraocular pressure in the aging population. Invest Ophthalmol Vis Sci. 1999;40(12):2439-2442.

18. Barkana Y, Anis S, Liebmann J, et al. Clinical utility of intraocular pressure monitoring outside of normal office hours in patients with glaucoma. Arch Ophthalmol. 2006;124(6):793-797. doi:10.1001/ archopht.124.6.793

19. Kim HJ, Cho BJ. Long-term effect of latanoprost on central cornea thickness in normal tension glaucoma. J Ocul Pharmacol Ther. 2011;27(1):73-76. doi:10.1089/jop.2010.0071

20. Zheng X, Wang Y, Zhao Y, et al. Experimental evaluation of latanoprost-induced changes in biomechanical behavior of ex-vivo rabbit corneas. Curr Eye Res. 2019;44(1):19-24. doi:10.1080/ 02713683.2018.1516781 


\section{Publish your work in this journal}

Clinical Ophthalmology is an international, peer-reviewed journal covering all subspecialties within ophthalmology. Key topics include: Optometry; Visual science; Pharmacology and drug therapy in eye diseases; Basic Sciences; Primary and Secondary eye care; Patient Safety and Quality of Care Improvements. This journal is indexed on PubMed

Submit your manuscript here: https://www.dovepress.com/clinical-ophthalmology-journal
Central and CAS, and is the official journal of The Society of Clinical Ophthalmology (SCO). The manuscript management system is completely online and includes a very quick and fair peer-review system, which is all easy to use. Visit http://www.dovepress.com/ testimonials.php to read real quotes from published authors. 